\title{
Utilização do questionário PSCHO na avaliação do clima de segurança do paciente:
}

\section{uma revisão integrativa}

\author{
Use of the PSCHO questionnaire in the patient safety climate assessment: an integrative review \\ Uso del cuestionario PSCHO en la evaluación climática de seguridad del paciente: una revisión
} integrativa

Recebido: 23/03/2021 | Revisado: 29/03/2021 | Aceito: 30/03/2021 | Publicado: 10/04/2021

Thaiane Almeida Silva

ORCID: https://orcid.org/0000-0002-2971-5937 Universidade Federal do Paraná, Brasil

E-mail: thaiane.pol@ hotmail.com

Patricia Cardoso Portella

ORCID: https://orcid.org/0000-0002-4445-2485 Universidade Federal do Paraná, Brasil

E-mail: patriciacardosoportella@gmail.com

Karla Crozeta Figueiredo

ORCID: https://orcid.org/0000-0003-3544-5643 Universidade Federal do Paraná, Brasil

E-mail: karla.crozetafigueiredo@gmail.com Lillian Daisy Gonçalves Wolff

ORCID: https://orcid.org/0000-0002-5152-4811 Universidade Federal do Paraná, Brasil

E-mail:1dgwolff@gmail.com

Eliane de Fatima Lima

ORCID: https://orcid.org/0000-0001-5128-3715

Universidade Federal do Espírito Santo, Brasil

E-mail: lianelima66@gmail.com

\begin{abstract}
Resumo
Objetivo: Identificar na literatura estudos que utilizaram o questionário Patient Safety Climate in Healthcare Organizations (PSCHO) na avaliação do clima de segurança do paciente em serviços de saúde hospitalares. Método: Trata-se de uma revisão integrativa da literatura, definida como pesquisa concisa em área específica. A busca e seleção dos estudos foi realizada por duas revisoras, de forma independente nas bases de dados LILACS, MEDLINE, CINAHL e WoS. Os resultados foram apresentados por meio do fluxograma PRISMA. Resultados: Foram recuperados na busca seis artigos, dos quais quatro foram incluídos e analisados. Os estudos foram realizados na China e nos Estados Unidos e as dimensões "medo de culpabilização e punição" e "medo da vergonha" sobressaíram de forma negativa nos estudos chineses. O PSCHO apresentou similaridades quando comparado a outros estudos que avaliaram o clima de segurança do paciente utilizando outros questionários de clima. Conclusão: Os resultados demonstraram que o PSCHO é útil para avaliar o clima de segurança do paciente. A ausência de estudos brasileiros utilizando o questionário indica uma potencialidade de sua aplicação em novas pesquisas, como estratégia para a melhoria da qualidade e promoção da segurança do paciente nas Organizações de Saúde.
\end{abstract}

Palavras-chave: Segurança do paciente; Cultura organizacional; Inquéritos e questionários.

\begin{abstract}
Objective: To identify in the literature studies that used the Patient Safety Climate in Healthcare Organizations (PSCHO) questionnaire to assess the patient safety climate in hospital health services. Method: This is an integrative literature review, defined as a concise survey in a specific area. The search and selection of studies were carried out by two reviewers, independently in the databases LILACS, MEDLINE, CINAHL, and WoS. The results were presented using the PRISMA flowchart. Results: Six articles were retrieved in the search, of which four were included and analyzed. The studies were carried out in China and the United States and the dimensions "fear of blame and punishment" and "fear of shame" stood out negatively in Chinese studies. The PSCHO showed similarities when compared to other studies that evaluated the patient's safety climate using other climate questionnaires. Conclusion: The results demonstrated that the PSCHO is useful for assessing the patient's safety climate. The absence of Brazilian studies using the questionnaire indicates a potential for its application in new research, as a strategy for improving the quality and promoting patient safety in Health Organizations.
\end{abstract}

Keywords: Patient safety; Organizational culture; Surveys and questionnaires. 


\begin{abstract}
Resumen
Objetivo: Identificar en la literatura estudios que utilizaron el cuestionario Clima de seguridad del paciente en las organizaciones sanitarias (PSCHO) para evaluar el clima de seguridad del paciente en los servicios de salud hospitalarios. Método: Se trata de una revisión integradora de la literatura, definida como una encuesta concisa en un área específica. La búsqueda y selección de estudios fue realizada por dos revisores, de forma independiente en las bases de datos LILACS, MEDLINE, CINAHL y WoS. Los resultados se presentaron utilizando el diagrama de flujo PRISMA. Resultados: Se recuperaron seis artículos en la búsqueda, de los cuales cuatro fueron incluidos y analizados. Los estudios se realizaron en China y Estados Unidos y las dimensiones "miedo a la culpa y al castigo" y "miedo a la vergüenza" destacaron negativamente en los estudios chinos. El PSCHO mostró similitudes en comparación con otros estudios que evaluaron el clima de seguridad del paciente mediante otros cuestionarios climáticos. Conclusión: Los resultados demostraron que el PSCHO es útil para evaluar el clima de seguridad del paciente. La ausencia de estudios brasileños que utilicen el cuestionario indica un potencial para su aplicación en nuevas investigaciones, como una estrategia para mejorar la calidad y promover la seguridad del paciente en las Organizaciones de Salud.
\end{abstract}

Palabras clave: Seguridad del paciente; Cultura organizacional; Encuestas y cuestionarios.

\title{
1. Introdução
}

O avanço do conhecimento vem acompanhado de desafios e demanda constante desenvolvimento de pesquisas científicas. É neste sentido que a busca pela qualidade do atendimento nos estabelecimentos de saúde incentiva a investigação sobre a segurança do paciente. No Brasil, o Programa Nacional de Segurança do Paciente estimula o compromisso com a qualidade dos serviços e a promoção da cultura de segurança do paciente (Brasil, 2013). Desta forma, as pesquisas na área buscam investigar valores e comportamentos entre as equipes no que se refere à prestação de cuidado seguro, abrangendo aspectos institucionais, coletivos e individuais (Pagani, Crozeta \& Crisigiovanni, 2019).

$\mathrm{Na}$ busca de avaliar a cultura de segurança do paciente, os questionários sobre clima de segurança têm sido amplamente empregados, com a finalidade de extrair a percepção que os profissionais têm a partir das experiências vividas no serviço de saúde. Este conjunto de percepções define o clima, uma parte mensurável da cultura de segurança, mas não a totalidade dela. Contudo, avaliar o clima possibilita identificar aspectos da conduta organizacional pertinentes à cultura de segurança (Carvalho et al., 2017).

O modo como os processos são conduzidos na organização expressam sua cultura, e é neste ponto que os termos clima e cultura se diferenciam. A cultura de segurança é um subproduto da cultura organizacional, sendo multinivelar, profunda e extensa. A cultura organizacional, por sua vez, é o conjunto de valores e atitudes perpassados individualmente pelos membros da organização. Logo, incorporar uma cultura de segurança envolve o compromisso de todos que a compõem (Abreu et al., 2019; Schein., 2001).

Avaliar o clima de segurança do paciente é um componente fundamental para a produção do conhecimento nas ciências da saúde, portanto, para que essa pesquisa avaliativa seja criteriosa, os questionários utilizados para mensurá-lo devem possuir acurácia psicométrica e estarem coesos com a temática a que se destinam, trazendo desta forma robustez aos dados (Benzer, Meterko \& Singer, 2017).

Esses questionários constituem-se ferramentas de pesquisa que possuem a vantagem de obter dados de forma rápida com vista a acessar variáveis que se queira avaliar, possibilitando a adoção de medidas e a realização de acompanhamento de planos de ação previamente implantados no serviço, bem como formulação de novas políticas (Vasconcelos, Arruda, Freire, \& Carvalho, 2018).

Dentre a gama de questionários autoaplicáveis utilizados para avaliar o clima de segurança, o Patient Safety Climate in Healthcare Organizations (PSCHO) é uma ferramenta que possibilita a obtenção de aspectos da cultura de segurança, revelando como os funcionários reproduzem e compartilham os elementos desta e fornecendo subsídios aos gestores sobre aspectos institucionais que precisam ser trabalhados, uma vez que a cultura de segurança do paciente é indissociável da cultura organizacional (Cunha \& Guirardello, 2018). 
O PSCHO é organizado em dimensões que representam três níveis de cultura de segurança: Organização, Unidade de Trabalho e Interpessoal. Após transitar por versões de 38 a 45 itens, adaptado às particularidades de cada contexto, é composto atualmente por 51 itens, sendo 45 relacionados à avaliação do clima e seis questões de caracterização profissional. O questionário foi desenvolvido a partir da lógica das Organizações de alta confiabilidade como as do setor da aeronáutica e suas ramificações, pela Agency for Healthcare Research and Quality (ARQH) dos Estados Unidos, visto a preocupação com a avaliação criteriosa de atividades complexas, nas quais o erro pode produzir danos irreparáveis (Singer et al., 2007).

O questionário foi recentemente traduzido e validado para o cenário brasileiro e está disponível para a realização de pesquisas que objetivem obter o clima de segurança do paciente, comparar os aspectos da cultura de segurança entre organizações de diferentes naturezas administrativas, bem como realizar estudos multicêntricos por todo o território nacional (Cunha \& Guirardello, 2018).

Diante do exposto, esta revisão teve como objetivo identificar na literatura estudos que utilizaram o Patient Safety Climate in Healthcare Organizations (PSCHO) na avaliação do clima de segurança do paciente em serviços de saúde hospitalares.

\section{Metodologia}

Trata-se de uma revisão integrativa, que consiste em uma síntese das informações disponíveis em dado momento, sobre um problema específico, de forma objetiva e reproduzível (Mendes, Silveira \& Galvão, 2008). Esse tipo de pesquisa apresenta um método rigoroso de busca e seleção de estudos, com avaliação da relevância e da validade dos resultados evidenciados, coleta, síntese e interpretação dos dados. Desta forma, elaborou-se um protocolo, a fim de garantir o rigor do processo de pesquisa, que dispunha dos seguintes componentes: pergunta de revisão, critérios de inclusão e exclusão, estratégias para a busca, orientação para a seleção do material, análise e síntese dos dados.

Para definição da questão de pesquisa, utilizou-se o acrônimo PCC (População, Conceito e Contexto), sendo respectivamente $\mathrm{P}$ = "Estudos de avaliação de clima de segurança do paciente", $\mathrm{C}$ = "Avaliação do clima de segurança do paciente" e $\mathrm{C}=$ "Serviços hospitalares". A questão de pesquisa foi assim definida: "Como e em quais contextos hospitalares o questionário PSCHO tem sido utilizado?"

Para identificar os estudos acerca do assunto, realizou-se, em outubro de 2020, uma busca nas bases Literatura Latinoamericana e do Caribe em Ciências da Saúde (LILACS), Medical Literature Analysis and Retrieval System Online (MEDLINE), Cumulative Index to Nursing and Allied Health Literature (CINAHL), SCOPUS e Web of Science (WoS). Utilizou-se a combinação dos seguintes descritores e operadores booleanos: "Patient Safety" OR "Organizational Culture" AND PSCHO, e "Segurança do Paciente" OR “Cultura Organizacional” AND PSCHO.

Para a inclusão dos artigos foram aplicados os seguintes critérios: estudos originais, no formato de artigos, que utilizaram o PSCHO para mensurar o clima de segurança do paciente em unidades hospitalares e/ou entre equipes multiprofissionais de unidades hospitalares e/ou entre hospitais, publicados em inglês, espanhol ou português a partir de 2007 (ano de publicação do PSCHO) até o ano de 2020. Os critérios de exclusão contemplaram artigos de tradução, adaptação transcultural e validação, e estudos de comparação entre o PSCHO e outros questionários. As buscas foram realizadas, de forma independente, por duas pesquisadoras (TAS; PCP). A seleção dos estudos foi feita a partir da análise dos títulos, resumos e textos completos das publicações.

Ao realizar um teste piloto nas bases selecionadas, notou-se escassez de estudos que utilizaram a ferramenta. Em seguida foram elaboradas oito estratégias de busca. Optou-se por ampliar o número inicial de estratégias a fim de viabilizar que a busca contemplasse o maior número de estudos possíveis que utilizaram o questionário PSCHO em ambientes hospitalares. No entanto, verificou-se repetitividade dos resultados retornados pelas diferentes estratégias nas bases selecionadas. Por fim, 
optou-se por utilizar apenas uma estratégia de busca, em Inglês e Português, por contemplarem todos os estudos encontrados nas demais buscas. O detalhamento da estratégia de busca e seleção dos estudos está descrito na Tabela 1.

Tabela 1 - Estratégia detalhada de pesquisa e seleção.

\begin{tabular}{l}
\hline Palavra-chave e MeSH \\
\hline$[\mathrm{MeSH}]$ "Patient Safety" \\
{$[\mathrm{MeSH}]$ "Organizational Culture" } \\
[Keyword] "PSCHO" \\
\hline Estratégias de Busca \\
\hline [Inglês] (("Patient Safety" OR "Organizational Culture") AND PSCHO) \\
[Português] (("Segurança do Paciente" OR "Cultura Organizacional”) AND PSCHO) \\
\hline Bases de Dados pesquisadas \\
\hline Cumulative Index to Nursing and Allied Health Literature (CINAHL), Medical Literature Analysis and Retrieval \\
System Online (MEDLINE), Latin American \& Caribbean Health Sciences Literature (LILACS), SCOPUS e Web of \\
Science (WoS).
\end{tabular}

Fonte: Autores.

A organização dos dados foi realizada por meio do software Mendeley ${ }^{\circledR}$ e a extração e categorização destes foi realizada pela primeira revisora e corrigida pela segunda revisora, em planilha Excel ${ }^{\circledR}$. Foram extraídos os dados referentes aos autores e ano de publicação, tipo de estudo, amostra, objetivos e principais resultados. A análise qualitativa realizou-se por meio de descrição e discussão dos resultados apresentados pelos estudos selecionados. Este estudo não envolveu seres humanos, estando de acordo com os aspectos éticos.

\section{Resultados}

Foram identificados 25 estudos nas bases de dados selecionadas. Após a remoção dos duplicados nas bases foi realizada a leitura do título e resumo de seis estudos, dos quais dois foram excluídos por serem, respectivamente, um estudo de construção de uma forma mais curta do PSCHO e um de tradução e adaptação transcultural. Por fim, foram selecionados quatro estudos originais para análise. A busca detalhada está ilustrada na Figura 1. 
Figura 1 - Etapas de seleção dos estudos nas bases de dados. Curitiba, PR, Brasil, 2020.
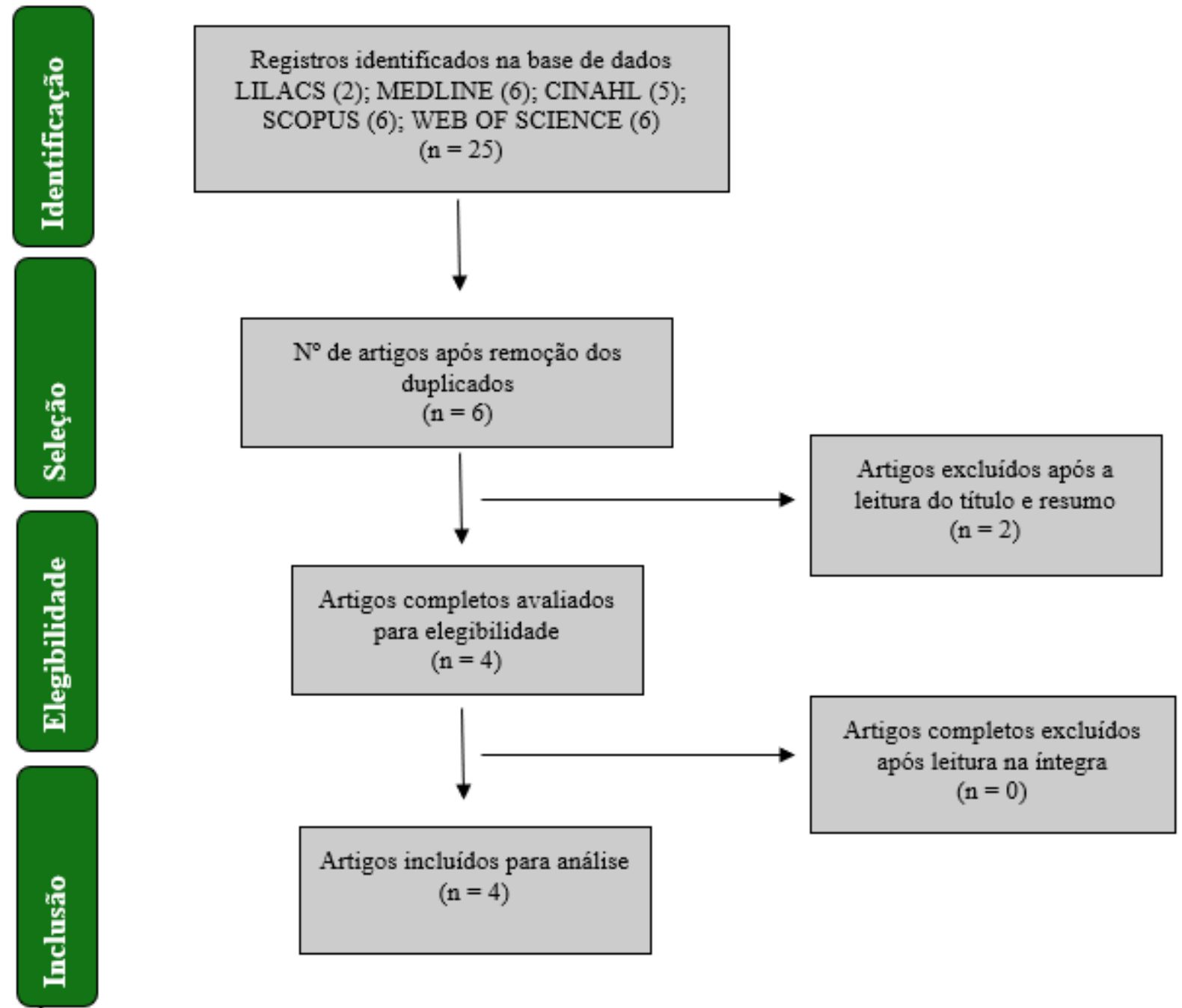

Fonte: Autoras, adaptado de Moher, Liberati \& Tetzlaff (2009).

Os resultados trouxeram dois estudos estadunidenses (E1 e E2) e dois estudos chineses (E3 e E4). Quanto à metodologia, todos os estudos fizeram uso do método descritivo transversal e utilizaram abordagem quantitativa de análise dos dados. Foram encontradas algumas similaridades entre os estudos em relação ao quantitativo amostral, à categoria profissional dos participantes e a tipos de unidades hospitalares nas quais o PSCHO foi aplicado. As características detalhadas dos estudos selecionados estão ilustradas na Tabela 2. 
Tabela 2 - Características dos estudos selecionados.

\begin{tabular}{|c|c|c|c|c|c|c|}
\hline Estudo & Ano & Tipo do estudo & Local & Tamanho amostral & Setores & Funcionários \\
\hline $\begin{array}{l}\text { El - } \\
\text { Hartmann et al }\end{array}$ & 2009 & Descritivo transversal & Estados Unidos & 4.625 & $\begin{array}{l}\text { Centro cirúrgico, SRP } \\
\text { UTIs e unidades de PA }\end{array}$ & $\begin{array}{l}\text { Gestores, chefes de } \\
\text { departamento, médicos } \\
\text { e demais funcionários }\end{array}$ \\
\hline $\begin{array}{l}\text { E3 - } \\
\text { Zhou et al }\end{array}$ & 2015 & Descritivo transversal & China & 1.272 & $\begin{array}{l}\text { Departamentos administra- } \\
\text { tivos e clínicos }\end{array}$ & $\begin{array}{l}\text { Gestores, chefes de } \\
\text { departamento, médicos } \\
\text { enfermeiros e demais } \\
\text { funcionários }\end{array}$ \\
\hline $\begin{array}{l}\text { E4 - } \\
\text { Zhou et al }\end{array}$ & 2018 & Descritivo transversal & China & 4.121 & $\begin{array}{l}\text { Medicina interna, unidade } \\
\text { cirúrgica, obstétrica, gine- } \\
\text { cológica, pediátrica, anes- } \\
\text { tesiologia, centro cirúrgico, } \\
\text { UTI, departamento auxilia } \\
\text { clínico }\end{array}$ & $\begin{array}{l}\text { Gestores, chefes de } \\
\text { departamento, médicos, } \\
\text { enfermeiros, técnicos } \\
\text { em saúde, auxiliares } \\
\text { cínicos }\end{array}$ \\
\hline
\end{tabular}

Fonte: Autores.

Os resultados foram compilados e classificados de acordo com o tema (Tabela 3).

Tabela 3 - Divisão temática dos fatores que interferem no clima de segurança do paciente.

\begin{tabular}{|c|c|c|}
\hline Temas & $\mathbf{N}$ & Referência \\
\hline Comunicação ineficaz & 1 & [E1] \\
\hline Culturas hierárquicas & 1 & {$[\mathrm{E} 3][\mathrm{E} 4]$} \\
\hline Cultura de culpa e punição & 2 & [E3][E4] \\
\hline Falta de percepção sobre os riscos & 1 & [E1] \\
\hline Falta de recursos organizacionais para segurança & 1 & [E3] \\
\hline Comportamentos inseguros & 1 & {$[\mathrm{E} 1][\mathrm{E} 2]$} \\
\hline Insegurança psicológica & 1 & [E2] \\
\hline
\end{tabular}

Fonte: Autores.

O PSCHO foi aplicado em unidades cirúrgicas (salas de cirurgia e salas de recuperação pós-anestésica - SRPA) e Unidades de Terapia Intensiva (UTIs) (E1), contudo, sem especificação quanto ao público atendido, se neonatal, pediátrico ou adulto; Unidades de pronto atendimento (PA) e Unidade de quimioterapia (E2); departamentos administrativos (E3); Ginecologia e obstetrícia, pediatria, anestesiologia e departamentos auxiliares clínicos (E4).

As amostragens foram de caráter multiprofissional e intersetorial, envolvendo funcionários desde a alta gestão até a assistência, reportados os últimos como funcionários da linha de frente. Apenas um estudo (Zhou et al., 2018) identificou todas as classes profissionais amostradas, os demais dividiram a análise em três categorias, sendo estas 1) gestores e chefes de departamentos; 2) médicos e 3) demais funcionários. Os resultados evidenciaram a utilização exclusiva da abordagem quantitativa $(n=4 ; 100 \%)$ para análise das variáveis do questionário autoaplicável, tanto na verificação do clima quanto da cultura de segurança do paciente em organizações hospitalares.

Apenas o E1 utilizou outro instrumento de avaliação de cultura organizacional, além do questionário autoaplicável PSCHO. Os autores buscaram entender em que nível as características da cultura organizacional afetam o clima de segurança 
em hospitais Veterans Affair (VA) nos Estados Unidos. A amostra de hospitais foi obtida mediante estratificação da amostra inicial em categorias (High, Medium, Low), permitindo uma seleção diversificada de hospitais de acordo com o nível de segurança que apresentam.

Além de características físicas e dos recursos humanos do hospital coletadas, um mix dos casos dos pacientes foi obtido por meio do uso do software PSI. Concomitantemente à aplicação do PSCHO, utilizado como instrumento principal, foi aplicado o instrumento de Zammuto e Krakower (1991) a um terço dos indivíduos, de como forma complementar, a fim de obtenção de características globais da cultura organizacional.

Ademais, foi utilizado um modelo linear hierárquico a fim de investigar as relações entre percepções dos funcionários sobre a segurança expressada em comportamentos e crenças, como também suas suposições em relação à cultura organizacional local. O software utilizado para as análises foi o STATA.

O estudo observou que os locais com maior adesão à cultura de grupo e à cultura empreendedora demonstraram índices positivos de clima de segurança ( $\beta=-0.30 \mathrm{e}-0.48$, respectivamente, $p<.001 \mathrm{em}$ ambos), ao contrário daqueles que aderem a culturas hierárquicas, que tendem para um clima de segurança mais fragilizado $(\beta=0.24, p<.001)$.

Também realizado em hospitais Veterans Affair (VA), o E2 objetivou identificar se o clima de segurança do paciente era melhor estabelecido nas unidades de cuidado pós-anestésico e nas salas de cirurgia, em relação aos demais setores hospitalares. Assim como no primeiro estudo (E1), os hospitais intensivistas escolhidos foram classificados conforme um critério de qualidade, divididos em quatro categorias e escolhidos aleatoriamente por categoria, para constituírem a amostra a ser pesquisada. Após aplicação do PSCHO, foram atribuídos pesos às respostas, foi utilizado o Teste $t$ de Student a fim de comparar respostas problemáticas entre as dimensões do PSCHO, bem como análise de regressão linear para as respostas problemáticas com variáveis dependentes.

O software SAS® foi empregado para análise estatística dos dados. Dentre todos os questionários, $n=324(7,74 \%)$ foram originados das unidades de cuidado pós-anestésico e das salas de operação. Pode ser verificado que, apesar da complexidade e do alto risco desses setores, a cultura de segurança era, de modo geral, muito semelhante a do restante do hospital.

Contudo, após a análise de cada item do PSCHO, individualmente, foi possível constatar maior fragilidade nas unidades cirúrgicas e nas salas de recuperação anestésica em relação aos demais setores, devido ao alto percentual de respostas problemáticas entre os itens relacionados com comportamentos inseguros dos colegas de equipe $(55.1 \%$ vs $43.2 \% ; p=.01)$, pouco interesse institucional para com a assistência (20.4\% vs $12.5 \%$; $p=.03$ ) e incompreensão da gerência sênior sobre os riscos relacionados ao atendimento $(28.3 \%$ vs $17.1 \% ; p=.01)$. Deste modo, evidenciou-se a necessidade de comunicação efetiva entre os gerentes e trabalhadores da linha de frente.

Destaca-se o apontamento dos autores ao descrever dentre os fatores limitantes do estudo, um diretamente relacionado ao PSCHO, ao afirmarem que apesar de ser uma ferramenta validada, não foi capaz de obter indicadores mais sutis para a cultura de segurança do paciente, como a hierarquia existente entre os funcionários médicos das unidades cirúrgicas, sendo este um cenário já conhecido pelos pesquisadores, devido a uma clara ordem hierárquica existente entre estes profissionais.

Dentre os estudos chineses, o primeiro estudo (E3) realizou algumas adaptações culturais no enunciado dos itens do PSCHO, como a troca do termo inglês "I" (eu) para o termo inglês "staff" (grupo ou pessoas que trabalham em uma organização). Posteriormente, a versão modificada teve sua confiabilidade testada mediante a análise do Alfa de Cronbach, testes de $\chi^{2}$ e análise de regressão multivariada. A necessidade das alterações foi justificada pela possível sensibilidade dos chineses à pessoalidade do termo "eu", podendo resultar em respostas inverídicas.

Trata-se de um estudo transcultural entre trabalhadores de hospitais chineses e estadunidenses. Dentre os 1.272 funcionários que responderam os questionários, em uma amostra de 1.268 em que se tem a informação de sexo, a maior parte 
$(\mathrm{n}=877,69,16 \%)$ eram mulheres, tendo trabalhado no hospital por 10 anos ou mais. A amostra foi constituída de gerentes $(\mathrm{n}=$ 47; 3,69\%), enfermeiros $(n=534 ; 41,98 \%)$, médicos $(n=505 ; 39,70 \%)$ e profissionais de outras profissões $(n=186 ; 14,62 \%)$.

O estudo demonstrou climas de segurança relativamente positivos em ambos os países, apesar disso, a dimensão "medo de culpabilização e punição" sobressaiu de forma negativa na China, correspondendo a n=385 (76.2\%) advindo dos médicos, $n=435(81.5 \%)$ dos enfermeiros e $n=31(65,96 \%)$ dos gerentes. Enquanto os mesmos itens desta dimensão não apresentaram relevância nos EUA. Em relação à dimensão "medo da vergonha", médicos, enfermeiros e gerentes apresentaram taxas respectivamente de $\mathrm{n}=231$ (45.8\%), $\mathrm{n}=214(40.07 \%)$ e $\mathrm{n}=16$ (36.04\%) nos hospitais chineses.

Os funcionários relataram que, quando a pressão aumenta, a gestão exige que o corpo de funcionários trabalhe mais rápido, deixando a assistência suscetível a erros. Os relatos sobre ter realizado prática insegura (34.33\%) ou ver o colega realizar algo que pudesse trazer danos ao paciente (38.54\%) apresentaram índices similares.

O estudo descreve a alta carga de trabalho como uma barreira potencial nos serviços de saúde chineses. Foi concluído que estudos deste tipo são muito necessários na China, a fim de estabelecer melhorias na segurança do paciente.

O segundo estudo chinês (E4), também conduzido por Zhou em colaboração com demais pesquisadores e realizado três anos após o primeiro, teve como objetivo avaliar o clima de segurança em hospitais chineses. A amostra incluiu nove departamentos e as variadas categorias entre 4.121. profissionais, incluindo médicos $n=1.593$ (38.66\%), enfermeiros $n=1.508$ (36.59\%), funcionários administrativos $\mathrm{n}=118$ (2.86) e técnicos da saúde $\mathrm{n}=382$ (9.27\%) que atuam em departamentos auxiliares. Dos participantes, a maioria possuía idade acima dos 45 anos $n=2.160$ (52.41) e predominantemente mulheres $n$ $=2.732(66.29 \%)$.

Para possibilitar a utilização do PSCHO, foram adicionados os itens "A equipe pode expressar livremente suas opiniões sobre a segurança do paciente" e "Analisamos acidentes ou eventos inesperados em tempo hábil" a fim de obter as percepções mais gerais sobre o clima de segurança do paciente. A versão modificada foi testada através do software SAS® a partir de um modelo de equação estrutural com os dados brutos. Ainda, foi realizada uma análise de multinível (nível 2), de modo a determinar a relação dos departamentos e o tipo de trabalho em cada dimensão do questionário. Os autores determinaram um índice $<3$, para considerar uma resposta como positiva, e $>3$, para uma resposta negativa (problemática).

As respostas válidas obtidas foram originadas dos setores de medicina interna, cirurgia, obstetrícia e ginecologia, pediatria, UTI, departamento de emergência, anestesiologia, salas de operação e departamento auxiliar clínico. Dentre os dados obtidos, percebeu-se mais uma vez um alto índice de respostas problemáticas na dimensão "medo de culpabilização e punição", $\mathrm{n}=2.670$ (64.79\%), e "medo da vergonha", $\mathrm{n}=841$ (20.41\%), presente em todas as categorias profissionais, com destaque entre os funcionários do departamento cirúrgico; enquanto que a dimensão "recursos organizacionais para segurança" obteve maior destaque na Pediatria (37.03\%) em relação aos outros departamentos. Quando comparada com os dados oriundos dos questionários aplicados aos gestores e chefes de departamento, a classe médica obteve maior índice de respostas negativas.

O estudo evidenciou diferenças nos índices de respostas problemáticas entre os departamentos pesquisados, demonstrando que alguns setores podem necessitar de maior atenção em relação à segurança do paciente bem como de técnicas de avaliação específicas conforme suas particularidades.

\section{Discussão}

Apesar do PSCHO ser um questionário que mede o clima de segurança do paciente, os autores utilizaram a terminologia cultura de segurança como sinônimo de clima de segurança ao reportarem seus resultados. Contudo, o clima de segurança é a junção das percepções sobre o estado no qual a Cultura se encontra, o ambiente de trabalho de forma ampla, desde a alta gestão até os processos de trabalho realizados em uma Organização de Saúde. Ao utilizar um questionário de clima 
de segurança, avalia-se, comumente na forma de escores positivos e negativos, as percepções sobre as dimensões da cultura de segurança do paciente (Russo, 2017; Santiago \& Turrini, 2015).

O caráter metodológico transversal de abordagem quantitativa é característico das pesquisas de clima de segurança, o que justifica o emprego unânime da metodologia encontrada nos estudos. No entanto, cabe salientar que estudos que aplicaram uma abordagem mista de análise de dados descrevem benefícios em utilizar o componente qualitativo em conjunto à abordagem quantitativa, especialmente para a pesquisa de cultura organizacional, visto que a triangulação de dados tem potencial para preencher as lacunas que os questionários autoaplicáveis por vezes apresentam (Russo, 2017; Creswell J \& Creswell D, 2018).

O PSCHO foi utilizado em diferentes cenários hospitalares, desde unidades de alto risco às de risco intermediário e baixo. Porém, já foi usado no último parágrafo obteve maior destaque nas unidades de cirurgia, bem como nas salas de recuperação anestésica, UTIs e unidades de pronto-atendimento. Quanto ao perfil dos pesquisados, a categoria médica esteve presente na totalidade dos estudos, juntamente com os gerentes e chefias departamentais, enquanto apenas um estudo descreveu as demais categorias profissionais como parte da amostra.

O uso do PSCH0 possibilitou evidenciar nestes cenários que, quanto mais hierárquica a cultura organizacional, mais apreensivos e inseguros os funcionários se tornam, impactando negativamente na segurança do paciente. Ao passo que as organizações que priorizam cultura de grupo ou empreendedora tendem a ter melhores resultados do que as que seguem modelos hierárquicos (Hartmann et al., 2009).

Características organizacionais como hierarquia, rigidez e poder centralizado, caracterizam organizações orientadas apenas para trabalho e processos, sendo, portanto, inflexíveis. A comunicação débil entre gestores e funcionários, controle e rigidez excessiva e verticalização nos processos de trabalho são fatores que impactam negativamente a segurança do paciente, visto que envolto neste meio o funcionário se vê pressionado no desenvolvimento de suas funções, comprometendo a qualidade da assistência (Cauduro, L. M. Sarquis, L. M. M. Sarquis, \& Cruz, 2015).

Demais fatores como comunicação ineficaz (Haytham et al., 2009), medo da culpabilização e vergonha frente ao erro (Zhou, Bundorf, Gu, He \& Xue, 2015; Zhou et al., 2018) foram itens que apresentaram alto índice de respostas problemáticas. Ao discutir sobre segurança do paciente é inegável a ênfase que se dá ao erro e à cultura de punição, instaurada em diversas organizações de saúde. A cultura punitiva fomentada pela falta de respaldo dos gestores leva o funcionário a não ocupar o seu lugar de fala, extinguindo as oportunidades de aprender com os erros. Urge, portanto, a necessidade de desenvolver a cultura justa, identificada como uma maneira de trazer, para os dois lados, justiça, ao reconhecer as responsabilidades pertinentes a funcionários e ao sistema (Lemos et al., 2018).

Os itens relacionados à falta de percepção dos riscos e pouco compromisso por parte da gerência obtiveram destaque. Esta variável engloba a verticalização dos processos de trabalho e comunicação frágil entre gestores, administradores e funcionários.

Demais estudos obtiveram resultados similares ao utilizar um outro instrumento de avaliação do clima de segurança e destacaram a direta relação entre a satisfação no trabalho com a segurança do paciente, o que indica a necessidade de melhoria nos processos de comunicação e envolvimento destes profissionais para a melhoria da qualidade do cuidado (Pagani, Crozeta \& Crisigiovanni, 2019; Souza, Rocha \& Mazzo, 2018).

As questões identificadas, caracterizadas como problemáticas, são fatores que vão na contramão do estabelecimento e fortalecimento de uma cultura justa, com priorização nos processos de trabalho em equipe, mitigação da cultura de punição, estímulo às relações de confiança imbricados na cultura Organizacional, na qual os funcionários se sintam motivados para notificar e reportar os erros ou situações de risco, com garantia de respaldo contra represálias (Reason, 2000; Marx, 2001).

\section{Conclusão}


Esta revisão possibilitou verificar que o questionário PSCHO foi pouco utilizado desde sua concepção. Foi possível observar sua similaridade com os demais questionários que têm sido largamente utilizados nas pesquisas de clima de segurança do paciente, visto constituir-se de dimensões da cultura organizacional de segurança do paciente. As pesquisas que o utilizaram puderam obter escores, negativos e positivos, que revelam a percepção dos membros da Organização de Saúde.

Apesar de o número reduzido de estudos encontrados e de não especificação das categorias profissionais amostradas pela maior parte dos estudos, as quais se configuram como limitação desta revisão integrativa, pode-se concluir que o PSCHO possibilitou observar os fatores que interferem na cultura de segurança do paciente nas unidades investigadas, ao ponderarem sobre a necessidade de fomentar culturas mais abertas às iniciativas individuais e concedendo assim espaços de fala aos funcionários, permitindo um trabalho coletivo mais coeso e com melhoria das relações entre chefias e funcionários. Em contrapartida, Organizações hospitalares altamente hierárquicas estão relacionadas a um maior medo de errar e estimulam a culpabilização individual, possuindo piores índices em relação ao clima de segurança do paciente.

O PSCHO constitui-se ferramenta para determinar o clima de segurança, visto que revela o atual estado da cultura de segurança da Organização. Outrossim, o questionário possibilita a realização de estudos transculturais e comparativos. Deste modo, recomenda-se a realização de novas pesquisas utilizando-o em outros cenários, como serviços ambulatoriais e atenção primária, visto que se relevou como uma nova possibilidade para as pesquisas de avaliação de clima de segurança do paciente, subsidiando assim a discussão e o avanço de práticas seguras.

\section{Referências}

Abreu, I. M., Rocha, R. C., Avelino, F. V. S. D., Guimarães, D. B. O., Nogueira, L. T., \& Madeira, M. Z. A. (2019). Cultura de segurança do paciente em centro cirúrgico: visão da enfermagem. Rev. Gaúcha Enferm. 40: e20180198.

Arboit, E. L., Bellini, G., Schütz, C. R., Moraes, M. C. S., Kinalski, S. S., \& Barcellos, R. A. (2020). Patient safety culture in the multiprofessional perspective. Research, Society and Development. 9(5).

Benzer, J. K., Meterko, M., \& Singer, S. J. (2017). The patient safety climate in healthcare organizations (PSCHO) survey: Short-form development. J Eval Clin Pract. 23:853-859.

Carvalho, R. E., Arruda, L P., Nascimento, N. K., Sampaio, R. L., Cavalcante, \& M. L., Costa, A. C. (2017). Assessment of the culture of safety in public hospitals in Brazil. Rev Lat Am Enfermagem. 2;25:e2849.

Cauduro, F. L. F., Sarquis, L. M., Sarquis, L. M. M., \& Cruz, E. D. A. (2015). Cultura de segurança entre profissionais de centro cirúrgico. Cogitare Enfermagem. 20(1).

Cunha, M. R. P. D., \& Guirardello, E. B. (2018). Patient Safety Climate in Healthcare Organizations: tradução e adaptação para a cultura brasileira. Rev. Gaúcha Enferm. 39:e20180010.

Creswell, W. J., \& Creswell, J. D. (2018). Research design: qualitative, quantitative, and mixed methods approaches (5a ed). Los Angeles: SAGE.

Gonzáles-Limas, W. R., Bastidas-Jurado, C. F., Figueiroa-Chaves, H. Á., Zambrano-Guerrero, C. A., \& Matabanchoy-Tulcán, S. M. (2018). Revisión sistemática de las concepciones de cultura organizacional. Univ. Salud. 20(2).

Hartmann, C. W., Meterko, M., Rosen, A.K., Zhao, S., Singer, S., \& Gaba, D.M. (2009). Relationship of Hospital Organizational Culture to Patient Safety Climate in the Veterans Health Administration. Medical Care Research and Review. 66(3).

Haytham, M. A., Kaafarani, M. D., Kamal, M. F., Itani, M. D., Rosen, A. K., Shibei, Z., Hartmann, C. W., Gaba, D. M. (2009). How does patient safety culture in the operating room and post-anesthesia care unit compare to the rest of the hospital? The American Journal Surgery. 198(70-75).

Lemos, G. C., Azevedo, C., Bernardes, M. F. V. G., Ribeiro, H. C. T. C., Menezes, A. C., \& Mata, L. R. F. (2018). A cultura de segurança do paciente no âmbito da enfermagem: reflexão teórica. Revista de Enfermagem do Centro-Oeste Mineiro. 8:e2600.

Marx, D. (2001). Patient safety and the 'just culture:' a primer for health care executives. Columbia University.

Ministério da Saúde (BR). Portaria $\mathrm{n}^{\circ}$ 529, de $1^{\circ}$ de abril de 2013: institui o Programa Nacional de Segurança do Paciente (PNSP) [internet]. Brasília: Ministério da Saúde.

Mendes, K. S., Silveira, R. C. C. P., \& Galvão, C. M. (2008). Revisão integrativa: método de pesquisa para a incorporação de evidências na saúde e na enfermagem. Texto \& contexto enfermagem. 17(4).

Moher, D., Liberati, A., Tetzlaff, J. (2009). Preferred reporting items for systematic reviews and meta-analyses: the PRISMA statement. Annals of internal medicine. 151(4). 
Research, Society and Development, v. 10, n. 4, e28210414200, 2021

(CC BY 4.0) | ISSN 2525-3409 | DOI: http://dx.doi.org/10.33448/rsd-v10i4.14200

Pagani, S., Crozeta, K. F., \& Crisigiovanni, A. B. R. (2019). Cultura de segurança do paciente: avaliação de enfermeiros. Rev Rene. $20:$ :e39782.

Reason, J. (2000). Human error: models and management. BMJ; 320(7237):768-70.

Russo, G.M. (2017). Diagnóstico da cultura organizacional: o impacto dos valores organizacionais no desempenho das terceirizações. Alta Books.

Santiago, T. H., \& Turrini, R. N. (2015). Cultura e clima organizacional para segurança do paciente em Unidades de Terapia Intensiva. Rev Esc Enferm USP. 49:123-130.

Schein, E. H. (2001). Guia de sobrevivência da Cultura corporativa. José Olympio.

Singer, S., Meterko, M., Baker, L., Gaba, D., Falwell, A., \& Rosen, A. (2007). Workforce Perceptions of Hospital Safety Culture: Development and Validation of the Patient Safety Climate in Healthcare Organizations Survey. HSR: Health Services Research. 42(5).

Souza, L. S., Rocha, F. R., Mazzo, L. L. (2018). Clima organizacional e ocorrência de acidentes com materiais perfurocortantes em um hospital público do Estado de São Paulo. Cad. Bras. Ter. Ocup. 26(1).

Vasconcelos, P. F., Arruda, L. P., Freire, V. E. C. S., \& Carvalho, R. E. F. L. (2018). Instruments for evaluation of safety culture in primary health care: integrative review of the literature. Public Health. 156.

Zhou, P., Bundorf, M. K., Gu, J., He, X., \& Xue, D. (2015). Survey on patient safety climate in public hospitals in China. BMC Health Services Research. 15(53).

Zhou, P., Bai, F., Tang, H-Q., Bai, J., Li, M-Q., \& Xue, D. (2018). Patient safety climate in general public hospitals in China: differences associated with department and job type based on a cross-sectional Survey. BMJ Open. 8(015604). 